\title{
Predicting the volumetric variation due to changes in suction, applied stress and swelling pressure
}

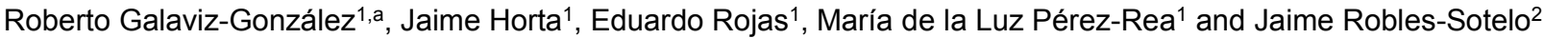

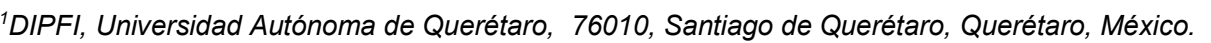

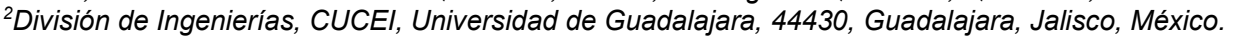

\begin{abstract}
The settlements produced by the load transmitted to the structure on expansive soils, and those calculated by the classical theories of soil mechanics, are different because the swelling pressure acts inversely to the applied stress. In this paper we describe a procedure to determine a volumetric variation coefficient by hydration $\left(\alpha_{h}\right)$ which considers the expansive soil behaviour. In order to do this, it is necessary to know the soil's initial water content, the swelling pressure, and the applied stress. Soil suction and swelling pressure were measured with filter paper technique and a mechanical oedometer, respectively. Unsaturated undisturbed samples of expansive soil were used. The water content was varied, starting from $0 \%$, with increments of $5.5 \%$ to reach $38 \%$. Furthermore, we present a set of curves that show the magnitude of the coefficient $\left(\alpha_{\mathrm{h}}\right)$ associated to a water content, and the relationship between applied stress and swelling pressure. The results show that the variation of the coefficient under different initial water contents ranges between $0 \%$ to $22 \%$. This is because water is strongly attracted by clay minerals, but this attraction decreases as water layers are father from the surface of clay minerals, thus decreasing its swelling potential.
\end{abstract}

\section{Introduction}

Lightweight buildings such as houses, can be damaged if they are built over expansive soils [1]. This is because water content variations induce volume strains on soils. This has led to overestimations and consequently to expensive foundations. This is because soil mechanics classical methodologies consider soils as if they were saturated, hence, they do not consider swelling pressure.

Some methods predict volumetric strains and optimize the design of foundations. These methods were proposed by Zeevaert [2], Nelson and Miller [3], Lytton [4], and ASTM D4546 [5]. Such methods require the swelling pressure as an input parameter. Therefore, various researchers have developed methods to predict the soil swelling pressure. Some of these methods use basic soil properties (indirect methods) and some others need a physical property which is measured in the laboratory (direct methods) [6]. However, prediction of volumetric changes rely heavily on the correct estimation of the swelling pressure.

This paper presents the determination of a coefficient $\left(\alpha_{\mathrm{h}}\right)$ associated to an initial water content $\left(\omega_{\mathrm{i}}\right)$ and the relationship between the applied stress and swelling pressure $\left(\sigma_{\text {app }} / \sigma_{\text {swe }}\right)$ measured on conventional laboratory equipment, which can be introduced into soilstructure interaction methods (SSI) in order to facilitate the prediction of soil settlement.

\section{Background}

\subsection{Expansive soils}

Expansive soils contain expansive clay minerals such as montmorillonite [7] that exhibit swelling behavior. This volumetric behavior has been recognized to compromise the stability of structures [8]. Light structures built on these soils usually contain structural damages as a result of the volumetric strains of the soils $[1,9]$. As these soils exhibit large volumetric strains, it is difficult to predict their behavior [8]. Swelling and expansive potential of soils mainly depend on the type and amount of clay minerals and the amount of water they absorb. When their water content increase, expansive soils swell affecting the structure stability [10].

\subsection{Swelling pressure estimation}

Some authors have proposed methodologies to estimate soil expansion that require the swelling pressure as an input parameter. Therefore, direct and indirect methods to estimate the swelling pressure have been proposed [6].

\footnotetext{
a Corresponding author: galaviz.gonzalez.r@gmail.com
} 
The swelling pressure is correlated with: liquid limit, plasticity index, the clay activity, water content, dry density and void ratio $[6,11]$.

Komornik and David (1969) proposed the following equation to correlate swelling pressure with water content, dry density and liquid limit for natural unaltered clayey soils:

$$
\log P_{s}=-2.132+0.0208 w_{L}+0.00665 \gamma_{d}-0.0269 w_{i}
$$

where: $P_{s}$ swelling pressure, $w_{L}$ liquid limit, $\gamma_{d}$ initial dry density, and $w_{i}$ initial water content.

Nayay and Christensen (1974) proposed a statistical relationship to predict swelling pressure as:

$$
P_{s}=\left(2.5 \times 10^{-1}\right)(P I)^{1.12}\left(C^{2} / w_{i}^{2}\right)+25
$$

where: $P_{s}$ swelling pressure, $P I$ plasticity index, $C$ clay content, and $w_{i}$ initial water content.

Lytton [4] proposed equations (16) and (17) to determine soil volumetric strains $\Delta V / V$.

$$
\begin{aligned}
& \sigma_{v}=\left(\frac{2}{3}\right) \sigma_{i} 10^{\frac{2 \varepsilon_{v}}{\gamma_{\sigma}(1-f)}}\left(\frac{h_{i}}{h_{f}}\right)^{\frac{\gamma_{h}}{\gamma_{\sigma}}}-\frac{\gamma_{\pi} z}{2} \\
& \varepsilon_{v}=f(\Delta V / V) \quad f=(1 / S r)-1
\end{aligned}
$$

where: $\sigma_{v}$ is the swelling pressure; $\sigma_{i}$ is the initial mean stress; $h_{i}$ and $h_{f}$ are the initial and final matric suction values respectively; $\gamma_{h}, \gamma_{\sigma}$, and $\gamma_{\pi}$ are compression indexes related to matric suction, mean stress and osmotic suction, respectively. $z$ is the thickness of the soil layer and $\mathrm{Sr}$ is the degree of saturation.

Erzin and Erol (2004) carried out standard constant volume swelling tests on a series of statically compacted clayey samples:

$$
\log P_{s}=-5.020+0.01383 P I+2.356 \gamma_{d}
$$

Rani and Rao (2009) studied the influence of the liquid limit, plastic index, water content and dry density on the swelling pressure of four types of clayey soils and proposed the following relationship:

$$
\begin{gathered}
\log P_{s}=-4.3341+0.0071 P I+0.0006 \log \gamma_{d}+\ldots \\
\ldots+51.2802 m_{c}+0.0037 S_{i}
\end{gathered}
$$

where: $P_{s}$ swelling pressure, $P I$ plasticity index, $\gamma_{d}$ dry density, $m_{c}$ water content, and $S_{i}$ initial surcharge pressure.

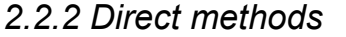

Direct methods usually involve laboratory procedures such as one-dimensional settlement measurement devices (oedometer) [12]. There are a number of procedures that can be reviewed in Shuai [12]. The most commonly ones are: 1) Free swell oedometer, 2) Loaded swell oedometer, and 3 ) constant volume oedometer.
In the free swell oedometer test, the soil specimen is brought in contact with water and allowed to swell freely with an applied load. Then the soil is gradually consolidated back to its original volume. The swelling pressure is defined as the necessary stress to consolidate the specimen back to its original volume [12].

In the loaded swell oedometer test, a number of identical specimens are subjected to different initial applied loads and allowed to swell freely. The resulting final volume strains are then plotted against the corresponding applied stresses. The stress corresponding to zero volume change is termed the swelling pressure [12].

In the constant volume test, a specimen is loaded and immersed in water. The specimen volume is maintained constant throughout the first part of the test by varying the applied load as required. This procedure is continued until there is no further tendency for swelling. The applied stress at this point is referred to as the "uncorrected" swelling pressure [12].

The free swell oedometer test usually results in an upper bound value for the swelling pressure. The loaded swell test gives the lowest value, and the constant volume method gives intermediate results [12].

After determining the swelling pressure, it is possible to estimate the volume strains with existing methodologies. However, the prediction of the volume strains depends heavily on the accuracy of the procedure used to estimate the swelling pressure. Baeza [13] made a comparison between the direct loaded swell oedometer and the indirect method proposed by Lytton, and concluded that the direct method is more feasible to use but, the indirect method provides consistent results.

\subsection{Volumetric variation prediction}

The total applied stress $\Delta \sigma$ to the soil-structure interface is defined as $\Delta \sigma=\sigma_{\text {swe }}-\sigma_{\text {app }}$. Here, we have three different possibilities:

Case a) When stress applied on soil mass is less than swelling pressure $\Delta \sigma_{a p p}<\sigma_{\text {swe }}$, then the settlements are negative. Here, the contact surface moves upwards [14].

Case b) The system is in equilibrium and the deformations at the contact surface are theoretically zero $\Delta \sigma_{\text {app }}=\sigma_{\text {swe }}[14]$.

Case c) When structural stress applied on soil mass is higher than swelling pressure $\Delta \sigma_{a p p}>\sigma_{\text {swe }}$, settlements will be positive but smaller in magnitude than in a nonexpansive soil [14]. In this case the coefficient $\alpha$ is determined by the theory of "Recompression by load" from Zeevaert [2]

When a load due to the weight of the building is placed on the foundation, the soil is recompressed and its elastic, plastic and visco-plastic components of deformation can be expressed as $[2,15]$ :

$$
\Delta \varepsilon_{c}=\Delta \varepsilon_{e o}+\Delta \varepsilon_{p o}+\Delta \varepsilon_{v p o}
$$

For unloading and total recompression $\Delta \sigma_{o i}$ the secant module $M_{c o}$ will be expressed as: 


$$
M_{c o}=M_{e o}\left(1+k_{p o}+k_{v p o}\right)=\frac{\Delta \varepsilon_{e o}}{\Delta \sigma_{c o}}\left(1+\frac{\Delta \varepsilon_{p o}}{\Delta \varepsilon_{e o}}+\frac{\Delta \varepsilon_{v p o}}{\Delta \varepsilon_{e o}}\right)
$$

For the case of an increment of stress $\Delta \sigma_{i}$ due to unloading or recompression, the secant modulus can be expressed as:

$$
M_{c i}=M_{e i}\left(1+k_{p i}+k_{v p i}\right)
$$

If expressions for $M_{c i}$ and $M_{c o}$ are considered, then, taking into account that parameters $k_{p o}$ and $k_{v p o}$ are proportional to each other, then for the level of stresses $\Delta \sigma_{i}$ the expression (10) can be derived to relate these parameters with the applied stresses for loading and unloading:

$$
\frac{k_{p i}}{k_{p o}} \cong \frac{\Delta \sigma_{i}}{\sigma_{c o}} \quad \frac{k_{v p i}}{k_{v p o}} \cong \frac{\Delta \sigma_{i}}{\sigma_{c o}}
$$

The recompression factor $\rho_{c}$ is defined as:

$$
\rho_{c}=\rho_{e i} \frac{1+\left(k_{p o}+k_{v p o}\right)\left(\Delta \sigma_{i} / \Delta \sigma_{c o}\right)}{1+\left(k_{p o}+k_{v p o}\right)}
$$

where: $\rho_{e i}$ represents the swelling factor, $\Delta \sigma_{c o}$ the increment of total stress and $\Delta \sigma_{i}$ is the partial stress increment.

The secant modulus for soil recompression is

$$
M_{c i}=\rho_{c i} \cdot M_{c o}
$$

and the volumetric variation coefficient $\alpha_{c i}$ for a layer of thickness $d_{i}$ is:

$$
\alpha_{c i}=M_{c i} \cdot d_{i}
$$

Nelson and Miller [3] proposed an equation for determining the expansion through the compressibility curve:

$$
\rho=\frac{C_{s} z_{i}}{\left(1+e_{o}\right)} \log \left(\frac{\sigma_{f}^{\prime}}{\sigma_{s c}^{\prime}}\right)
$$

where: $\rho$ represents the swelling deformation, $z$ is the layer thickness, $C_{s}$ is the swell index, $\sigma_{f}{ }_{f}$ stands for the final effective stress, $\sigma_{s c}^{\prime}$ is the corrected swelling pressure measured in a test at constant volume and $e_{o}$ is the initial void ratio.

Furthermore, Nelson and Miller [3] also proposed an expression to estimate the soil expansion, which involves the matric suction:

$$
\rho=\frac{z_{i}}{\left(1+e_{o}\right)}\left[c_{m} \Delta \log \left(u_{a}-u_{w}\right)+c_{t} \Delta \log \left(\sigma-u_{a}\right)\right]
$$

where: $c_{m}$ is a matric suction index, $c_{t}$ goes for the effective stress index, $\sigma$ is the net stress applied over the soil, $u_{a}$ represents the air pore pressure, $u_{w}$ is the water pore pressure, and is the $\left(u_{a}-u_{w}\right)$ matric soil suction.
Lytton [4] suggested that for the small increases in the volume change in constitutive surface (suction, mean stress, and volume) $(h, \sigma, V)$, the volumetric strain $\Delta V / V$ is linearly related.

$$
\begin{gathered}
\frac{\Delta V}{V}=\gamma_{h} \log \left(\frac{h_{f}}{h_{i}}\right)-\gamma_{\sigma} \log \left(\frac{\sigma_{f}}{\sigma_{i}}\right)-\gamma_{\pi} \log \left(\frac{\pi_{f}}{\pi_{i}}\right) \\
\gamma_{\sigma}=\frac{C_{c}}{1+e_{o}} \quad \gamma_{h}=\frac{\gamma_{\sigma}}{1+\left(\frac{h}{\theta(\partial h / \partial \theta)}\right)}
\end{gathered}
$$

where: $\Delta V / V$ is the volumetric strain; $h_{i}$ and $h_{f}$ are the initial and final matric suction values; $\sigma_{i}$ and $\sigma_{f}$ are the initial and final mean stresses; $\pi_{i}$ and $\pi_{f}$ are the initial and final osmotic suction values; $\gamma_{h}, \gamma_{\sigma}$, and $\gamma_{\pi}$ represent the compression indexes for matric suction, mean stress and osmotic suction, respectively. If we consider nil osmotic suction variations $\left(\pi_{i}=\pi_{f}\right)$ the last right hand side term of (16) is zero.

Finally, Arrieta and Sánchez [16] presented a set of equations to compute volumetric strains for any stress state under the foundation slab:

$$
\begin{gathered}
\varepsilon_{v}=\varepsilon_{v \max }\left[1-\frac{\log \left(\sigma_{e q} / \sigma_{v}\right)}{\log \left(\sigma_{e q} / \sigma_{o}\right)}\right] \\
\delta e=\varepsilon_{v} \cdot d_{i}
\end{gathered}
$$

where: $\varepsilon_{v m z x}$ is the maximum unitary volumetric strain, $\sigma_{o}$ represents the normal stress, $\sigma_{e q}$ stands for the expansive equilibrium stress and $d_{i}$ is the layer thickness.

\section{Materials and methods}

For this study, undisturbed soil samples were obtained from the region of Jurica in Santiago de Queretaro, Mexico, at depths between $0.60 \mathrm{~m}$ and $0.80 \mathrm{~m}$. Subsequently, tests to determine index properties were performed to establish the plasticity properties and grain size distribution curve of the soil samples.

For the determination of the volumetric variation coefficient, laboratory tests were performed with a mechanical oedometer using samples of unsaturated unaltered expansive soil with different initial water contents. A load of equal magnitude to that found at the site was placed on the specimen until the deformation was consistent. The saturation process began immediately and continued until the swelling deformation was developed, following the procedure outlined by method B in ASTM D4546 [5]. This swelling reflects the properties of expansive soil and depends solely on the initial water content.

Once the soil was saturated, typical consolidation tests were conducted, creating a hysteresis cycle in load and total unload [5]. The swelling pressure was measured in unaltered specimens with an initial water content rising in increments of $5.5 \%$ from $0 \%$ up to $38 \%$. These water 
contents represent a degree of saturation that rises in increments of $10 \%$ from $0 \%$ up to $70 \%$, which is because no volume increase neither swelling pressure acts with a degree of saturation equal to $70 \%$ was found. The initial strain caused by swelling soil is considered an important part of the elastic strain sustained by the material.

Suction tests with undisturbed specimens were also performed using filter paper technique (using Whatman ${ }^{\circledR}$ 42 filter paper) [17], in order to obtain relationship between water content (or degree of saturation) and soil suction (see Figure 1). The test started with dry samples ( $0 \%$ of the degree of saturation) whose degree of saturation was increased by steps up to nearly full saturation.

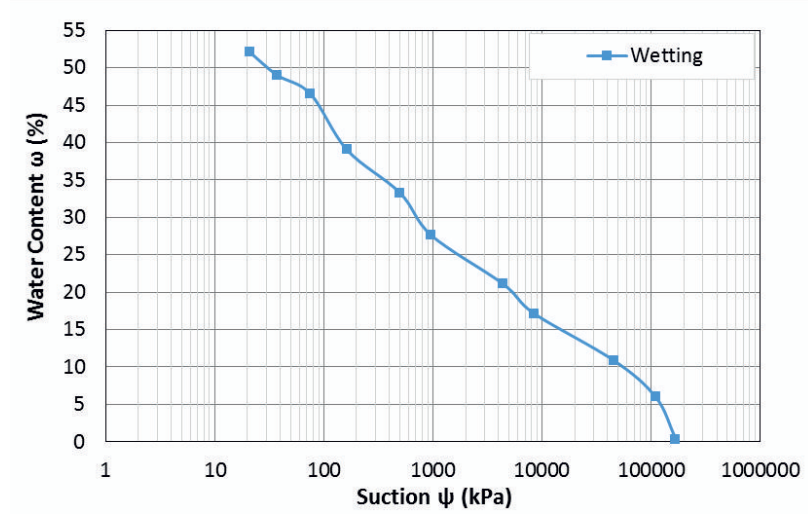

Figure 1. Soil-water retention curve (main wetting path).

\subsection{Estimation of volumetric variation coefficient by hydration $\alpha_{h}$}

The volumetric variation coefficient by hydration $\alpha_{h}$ depends on two types of stresses. The first one is soil suction increments $\Delta \sigma \psi$ and refers to variations of the soil moisture generating an effective stress relief that results in a swelling. The other stress is caused by applied external stresses $\Delta \sigma_{a p p}$ which are transmitted to the soil mass through the foundation.

Here, we propose a procedure to determine the volumetric variation coefficient $\alpha_{h}$ associated with an initial water content $\omega_{i}$ and the relationship between applied stresses and swelling pressure $\left(\sigma_{a p p} / \sigma_{\text {swe }}\right)$. Because a stress relief due to unloading is similar to a stress relief induced by soil hydration [18], the coefficient $\alpha_{h}$ is determined in the same way to that described by Zeevaert [2] for the coefficient $\alpha_{c i}$ in his theory "swelling by unloading".

Assuming that the relationship between the stresses induced by suction, and the corresponding deformations is linear, the maximum swelling secant modulus $M_{h o}$ due to changes in degree of saturation can be defined as:

$$
M_{h o}=\frac{\Delta \varepsilon_{h o}}{\Delta \sigma_{h o}}
$$

and the total strains due to variations in soil suction.

$$
\Delta \varepsilon_{h o}=a\left(\Delta \sigma_{h o}\right)^{C}
$$

combining (21) with (20) the secant modulus $M_{h o}$ is expressed as:

$$
M_{h o}=a\left(\Delta \sigma_{h o}\right)^{C-1}
$$

Similarly, partial stress relief can be expressed as:

$$
M_{h i}=a\left(\Delta \sigma_{h i}\right)^{C-1}
$$

combining (22) and (23):

$$
\frac{M_{h i}}{M_{h o}}=\left(\frac{\Delta \sigma_{h i}}{\Delta \sigma_{h o}}\right)^{C-1}
$$

Thus, the swelling factor by suction variations will be:

$$
\rho_{h}=\frac{M_{h i}}{M_{h o}}=\left(\frac{\Delta \sigma_{h i}}{\Delta \sigma_{h o}}\right)^{C-1}
$$

Assuming that applied stresses are proportional to suction [19], it follows that:

$$
\frac{\Delta \sigma_{h i}}{\Delta \sigma_{h o}}=\frac{\Delta \psi_{h i}}{\Delta \psi_{h o}}
$$

Therefore, stresses can be replaced by the soil suction in (25), where the swelling factor is:

$$
\rho_{h}=\left(\frac{\Delta \psi_{h i}}{\Delta \psi_{h o}}\right)^{C-1}
$$

In (27), $C$ is obtained from the plot in Figure 3 (swelling pressure against unitary strain), and represents its gradient:

$$
C=\frac{\log \left(\varepsilon_{f} / \varepsilon_{i}\right)}{\log \left(\sigma_{\text {swef }} / \sigma_{\text {swei }}\right)}
$$

Combining (26) and (27) the secant modulus for unitary strains $M_{h i}$ at a depth $z_{i}$ corresponding to the response to a partial stress relief induced by swelling is defined as:

$$
M_{h i}=\rho_{h i} \cdot M_{h o}
$$

The volumetric variation coefficient by hydration $\alpha_{h i}$ for each layer thickness $d_{i}$ is:

$$
\alpha_{h i}=M_{h i} \cdot d_{i}
$$

\section{Results and discussion}

In Figure 2 we show the relationship between the water content and swelling pressure obtained through an Oedometer tests. The relationship between swelling pressure $\sigma_{\text {swe }}$ and volumetric strain $\varepsilon$ can thus be inferred from Figure 3 .

Using a fitted equation to the experimental data in Figure 3 parameters $\varepsilon_{i}, \varepsilon_{f}, \sigma_{\text {swei }}$ and $\sigma_{\text {swef }}$ can be determined. 
They represent the minimum volumetric strain, maximum unitary volumetric, minimum swelling pressure and maximum swelling pressure respectively. Finally using (28) Zeevaert's index $C$ it can be computed as:

$$
\begin{array}{rrr}
\varepsilon_{i}= & 0.00617 \quad \varepsilon_{f}=0.28901 \\
\sigma_{\text {swei }}=1.05 \mathrm{kPa} & \sigma_{\text {swef }}=126.00 \mathrm{kPa} \\
C=\frac{\log \left(\varepsilon_{f} / \varepsilon_{i}\right)}{\log \left(\sigma_{\text {swef }} / \sigma_{\text {swei }}\right)}=0.8034 \frac{1}{\mathrm{kPa}}
\end{array}
$$

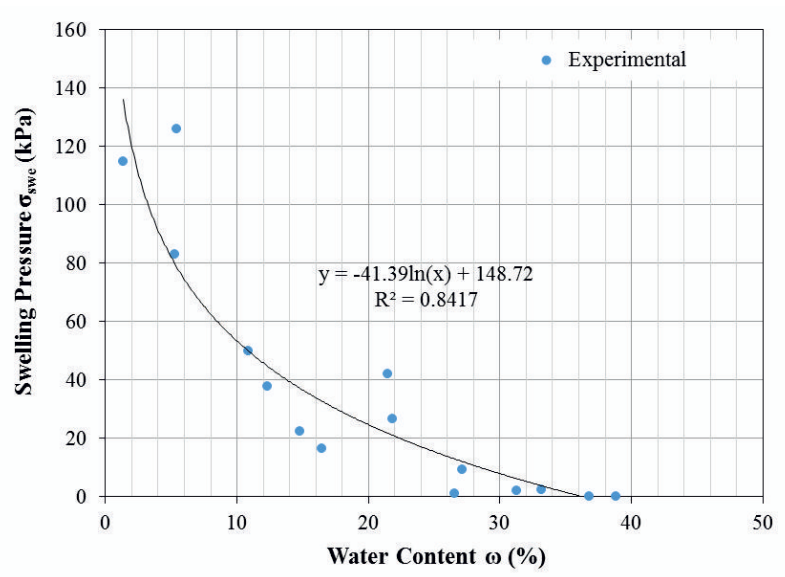

Figure 2. Relationship between water content and swelling pressure of Jurica's soil.

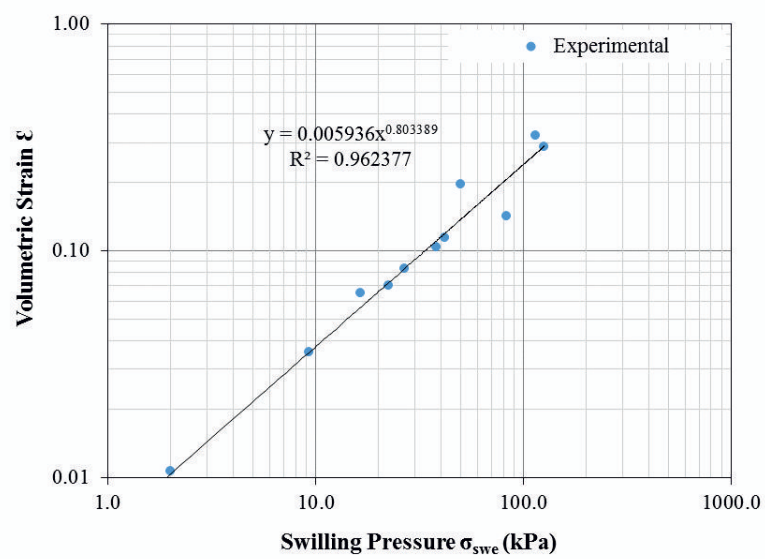

Figure 3. Relationship between swelling pressure and volumetric strain of Jurica's soil.

The maximum suction stress change induced by hydration $\Delta \psi_{h o}$, occurs when the soil is in an initial low water content and then nearly reaches full saturation. The following parameters can be obtained by inspection from Figure 3:

$$
\begin{array}{cr}
\omega_{i}=0.35 \% & \omega_{f}=52.07 \% \\
\psi_{o}=168,352.85 \mathrm{kPa} & \psi_{f}=21.08 \mathrm{kPa} \\
\Delta \psi_{h o}=\psi_{i}-\psi_{f}=168,331.77 \mathrm{kPa}
\end{array}
$$

According to (20) and (26), the secant modulus of unitary strain due to a total stress relief induced by water content variations $M_{h o}$ is:

$$
M_{h o}=\frac{\Delta \varepsilon_{h o}}{\Delta \sigma_{h o}}=\frac{\varepsilon_{f}-\varepsilon_{i}}{\psi_{i}-\psi_{f}}=1.68 \times 10^{-6} \frac{1}{k P a}
$$

Then, the value of partial suction changes $\Delta \psi_{h i}$ is computed by subtracting the initial suction $\psi_{i}$ from the next one $\psi_{i+l}$. This process will continue until the final suction $\psi_{f}$ is reached. The secant modulus for unitary strains related to a partial stress relief due to increase of water content of soil $M_{h i}$ is calculated with (29). With (27) the swelling factor $\rho_{h i}$ is computed. The process ends with the determination of the volumetric variation coefficient $\alpha_{h i}$.

Figure 4 shows the behavior of the variation volumetric coefficient $\alpha_{h}$. Starting with a water content of $0 \%$ and subjected to $1 \%$ increments. Notice that $\alpha_{h}$ shows the largest variations when the water content goes from $0 \%$ to $22 \%\left(8.21 \times 10^{-3}\right.$ a $\left.4.45 \times 10^{-3} \mathrm{~m} / \mathrm{kPa}\right)$. Whereas, when the water content varies from $22 \%$ to $55 \% \alpha_{h}$ remains practically unchanged. A polynomial fitting function has strong correlation $\left(\mathrm{R}^{2}=0.9926\right)$ with experimental data; this indicates that if the water content is above $0 \%, \alpha_{h}$ can be obtained with the procedure described earlier, however, lower values of the water content are difficult to find in common practice. It can be inferred that $\alpha_{h}$ can be computed accurately if the water content ranges between 0 to $100 \%$. For that reason, in order to determine the behavior of the variation volumetric coefficient $\alpha_{h}$ at different water content. Figure 4 also shows that, if the water content is between $0 \%$ and $22 \%$, the volumetric variation coefficient $\alpha_{h}$ takes its highest values (between $9.41 \times 10^{-3}$ and $4.45 \times 10^{-4} \mathrm{~m} / \mathrm{kPa}$ ). If the water content is above $22 \%, \alpha_{h}$ remains practically unchanged in any change of thereof.

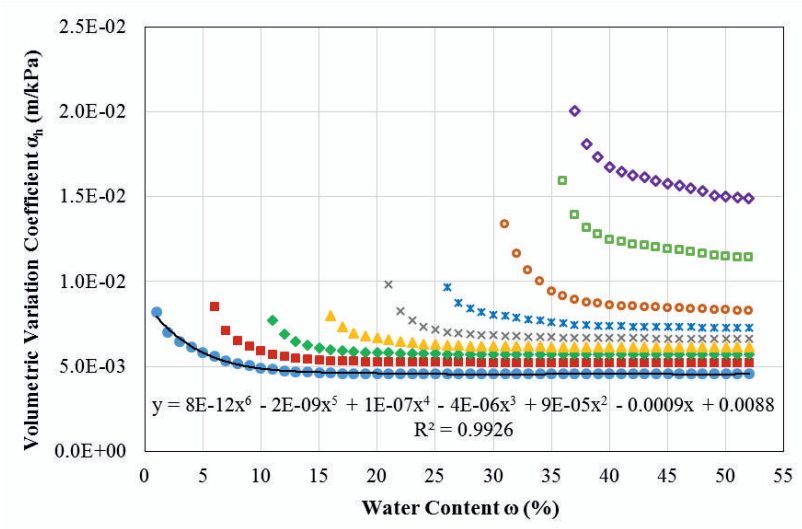

Figure 4. Behavior of volumetric variation coefficient with different initial water content.

Considering the previous ideas, the next step is to build up a data set for the ratio of applied stress and swelling pressure $\left(\sigma_{\text {app }} / \sigma_{\text {swe }}\right)$ ranging from 0 to 1 . The data set will be related to a curve with a specific initial water content $\omega$, this in turn represents a volumetric variation coefficient $\alpha_{h}$ in the x-axis.

With Figure 5 it can be noticed that the critical water content ranges from $0 \%$ to $22 \%$. Here $\alpha_{h}$ varies significantly, this is a difficult condition for the Jurica 
soil to meet as its water content ranges from $17 \%$ to $41 \%$ $[20,21]$.

Then, using Figure 5 is quite simple as the only parameters that are needed is the initial water content $\omega$, the applied stress $\sigma_{a p p}$, and the swelling pressure $\sigma_{\text {swe }}$ of soil. After that, the ratio $\left(\sigma_{a p p} / \sigma_{s w e}\right)$ is needed to be computed to input its value in the y-axis. Then an horizontal line is drawn to intersect the curve corresponding to the initial water content, and a vertical line is also drawn to cross the $\mathrm{x}$-axis where $\alpha_{h}$ will be found.

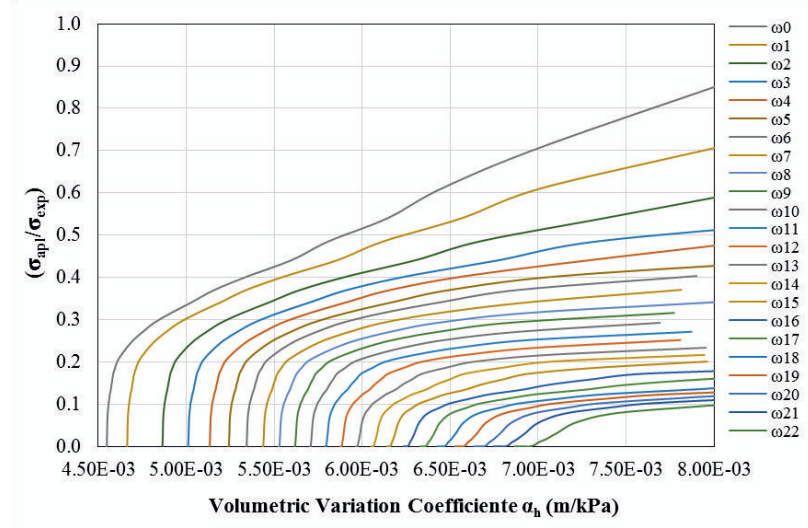

Figure 5. Volumetric variation coefficient associated to an initial water content (from $0 \%$ to $22 \%$ ), the relationship between applied stress and swelling pressure (from 0 to 1 ).

\section{Conclusions}

The volumetric variation coefficient shows its greatest fluctuations between $0 \%$ and $22 \%$ of the water content, but from $22 \%$ up to $55 \%$, the changes in its magnitude are not significant. This is because soil particles with water contents near zero experience strong attractions to water molecules. These forces tend to decrease as the water molecules move away from clay minerals surface decreases its swelling potential.

In determining the volumetric variation coefficient it is required to have good temperature humidity control and excellent testing conditions during any lab procedure. Small variations may induce great uncertainties.

The assumption that the swelling of a soil mass (induced by a stress relief by hydration) (changes in suction) has shown to be precise and provides an easy and simple tool that takes into account the soil expansive behavior in the analysis and design of foundations on these soils.

The results presented herein (such as Figure 5) cannot be generalized to the use of any expansive soil since the curves can be different for each soil due to a number of factors such as natural dispersion, different solid minerals, cracking planes, alteration due to sampling techniques, etc. Currently the authors are working with several other reported lab data coming from many different soils with similar characteristics. This, in order to establish relationships between them and get to use the results in common practice in the prediction of the coefficient.

\section{References}

1. $\square$ K. Kayabali, S. Demir, Can. Geotech. J. 48, 3, 354364 (2011)

2. $\square$ L. Zeevaert, Interección suelo-estructura de cimentación (D.F. Mexico: Limusa, 1980)

3. $\square$ J.D. Nelson, D. J. Miller, Expansive soils. Problems and preactice in foundation and pavement engineering (John Wiley \& Sons, Inc., 1992)

4. $\square$ R.L. Lytton, Geotechnical Special Publication. 2, 1827-1845 (1994)

5. ASTM D4546, Standard test methods for onedimensional swell or collapse of soils (2014).

6. $\square$ M.E. Zumrawi, University of Khartoum Engineering Journal. 3, 2, 35-39 (2013).

7. $\square$ H. King, $[$ On line $]$ Available: http://geology.com/articles/expansive-soil.shtml. [accesed: January 28, 2015].

8. J.P. Puppala, T. Manossuthikij, C.S. Chittoori, Engineering Geology. 164, 187-194 (2013).

9. $\square$ E. Jahangir, O. Deck, F. Masrouri, Comput. Geotech. 54, 16-32 (2013).

10. L.D. Jones, [On line]. Available: http://core.ac.uk/download/pdf/386982.pdf.2015. [accesed: March 25, 2015].

11. $\square$ A.T. Sudjianto, K.B. Suryolelono, A. Rifa, I.B. Mochtar, International Journal of Engenieering \& Technology. 11, 6, 142-148 (2011)

12. F. Shuai, Simulation of swelling pressure measurements on expansive soils (University of Saskatchewan, 1996)

13. A. J. Baeza, Diseño de zapatas continuas de cimentación sobre suelos expansivos mediante la metodología Lytton-Mitchel caso Querétaro (Universidad Autónoma de Querétaro, 2012)

14. M.L. Pérez-Rea, O. Cabrera, XXI Reunión Nacional de Mecánica de Suelos (Sociedad Mexicana de Mecánica de Suelos, 2002)

15. M.L. Pérez-Rea, T.I. Ayala, XIII Conferencia Panamericana de Mecánica de Suelos e Ingeniería Geotécnica (2007)

16. L. Arrieta, N.E. Sánchez, Mater. Cosntrucc., 53, 271-272 (2003)

17. $\square$ ASTM D5298, Standard test method for measurement of soil potential (suction) using filter paper (2010)

18. R. Galaviz-González, Predicción de asentamientos de cimentaciones superficiales mediante análisis de interacción suelo expansivo-estructura de cimentación (Universidad Autónoma de Querétaro, 2013)

19. M.L. Pérez-Rea, Succión y comportamiento esfuerzodeformación en suelos expansivos de Jurica y Tejeda (Universidad Autónoma de Querétaro, 1993)

20. T. López-Lara, Resistencia al esfuerzo cortante en arcillas expansivas de Jurica (Universidad Autónoma de Querétaro, 1996)

21. A.O. Cabrera, Determinación de la expansión volumétrica por hidratación en un suelo expansivo y su influencia en el analisis y diseño de cimentaciones (Universidad Autónoma de Querétaro, 2001) 